Investigación

\title{
CALIDAD DE LAS PRÁCTICAS FORMATIVAS EN LOS ÚLTIMOS SEMESTRES DE ENFERMERÍA DE LA UNIVERSIDAD CATÓLICA DE MANIZALES, COLOMBIA
}

\author{
THE QUALITY OF TRAINING PRACTICE IN THE LAST SEMESTERS OF NURSING \\ FROM THE CATHOLIC UNIVERSITY OF MANIZALES, COLOMBIA
}

\section{María Beatriz Escobar Escobar}

Enfermera. Doctora en pensamiento complejo (Multiversidad), Magister en enfermería MaternoInfantil, Universidad del Valle, Colombia

Especialista en docencia universitaria, Universidad de Caldas, Colombia

Especialista en Administración en Salud, Universidad Católica de Manizales, Colombia

Docente Asistente, Universidad del Quindío, Colombia

Integrante del grupo de investigación comunicación en salud Universidad Católica de

Manizales, Colombia

mbescobar@uniquindio.edu.co

http://orcid.org/0000-0002-0419-9032

\section{Nancy Álvarez Castaño}

Especialista en Administración de Servicios de Salud. Enfermera, Universidad de Caldas, Colombia

Docente Asistente, Universidad Católica de Manizales

Integrante del grupo de investigación comunicación en salud Universidad Católica de

Manizales, Colombia

nanalvarez@ucm.edu.co

https://orcid.org/0000-0001-6878-8031

\section{Stephania Rendón Caro}

Estudiante Enfermería

Universidad Católica de Manizales, Colombia

\section{Mariana Villegas Quintero}

Estudiante Enfermería

Universidad Católica de Manizales, Colombia

Valentina García Gallego

Estudiante Enfermería

Universidad Católica de Manizales, Colombia

\section{Lina Marcela Mantilla Sánchez}

Estudiante Enfermería

Universidad Católica de Manizales, Colombia

Correspondencia email: mbescobar@uniquindio.edu.co 
Escobar Escobar MB, Álvarez Castaño N, Rendón Caro S, Villegas Quintero M, García

Gallego V, Mantilla Sánchez LM

Artículo recibido el 6 de octubre de 2021. Aceptado en versión corregida el 26 de noviembre de 2021.

\title{
RESUMEN
}

INTRODUCCIÓN: La calidad de las prácticas formativas de enfermería deben ir acompañadas por un sistema de conocimientos genéricos, desarrollo permanente, y apoyo en la práctica clínica. OBJETIVO: Describir las condiciones de calidad de las prácticas formativas de los últimos semestres del programa de Enfermería de la Universidad Católica de Manizales con relación a los criterios básicos definidos por el Ministerio de Protección Social, el Ministerio de Educación Nacional, y los definidos por el programa de Enfermería en los años 2015 - 2017. METODOLOGÍA: Investigación descriptiva, retrospectiva, documental. Con la información suministrada por la literatura, el marco de regulación, los factores y las características del modelo de evaluación de las prácticas, entre los años 2015 al 2017, los criterios de inclusión fueron el formato de evaluación de prácticas de la Universidad del caso y exclusión que se emplearon. RESULTADOS: Muestran debilidades y fortalezas como que se descubre que la UCM cumple con todos los criterios de idoneidad como institución educativa, pero hay condiciones para mejorar. CONCLUSIONES: Realizar acompañamiento a las entidades como hospitales y centros de práctica, para obtener mejores escenarios de práctica.

Palabras clave: Educación en Enfermería, Docentes, Ciencias de la Salud, Prácticas Clínicas, Control de Calidad, DeCS.

\begin{abstract}
INTRODUCTION: The quality of nursing training practices must be accompanied by a generic knowledge system, permanent development, and support in clinical practice. OBJECTIVE: Describe the quality conditions of the training practices of the last semesters of the Nursing program of the Universidad Católica de Manizales in relation to the basic criteria defined by the Ministry of Social Protection, the Ministry of National Education, and those defined by the Nursing program in the years 2015 - 2017. Descriptive METHODOLOGY, retrospective, documentary film. With the information provided by the literature, the regulatory framework, the factors and characteristics of the internship evaluation model, between the years 2015 to 2017, the inclusion criteria were the internship evaluation format of the University of the case and exclusion that were used. RESULTS: Show weaknesses and strengths as it is discovered that the UCM meets all the criteria of suitability as an educational institution but there are conditions for improvement. CONCLUSIONS: Provide support to entities such as hospitals and practice centers, to obtain better practice scenarios.
\end{abstract}

Key words: Education, Nursing, Faculty, Health Sciences, Clinical Clerkship, Quality Control, DeCS. 
Calidad de las prácticas formativas en los últimos semestres de Enfermería...

\section{http://dx.doi.org/10.7764/Horiz_Enferm.32.3.306-321}

\section{INTRODUCCIÓN}

Dentro de la normatividad que regula el adecuado funcionamiento de las prácticas en salud, se enmarcan una serie de requisitos que identifican las debilidades y fortalezas de los involucrados en la relación docencia servicio, que den cuenta de los recursos administrativos, estructurales, tecnológicos y humanos y de oportunidades de atención, así como de la calidad en cada uno de los escenarios de práctica para el proceso enseñanza aprendizaje.

El modelo de evaluación de las condiciones de calidad de la relación docencia - servicio, estipulado en el Acuerdo 000003 de 2003, permite identificar el grado de desarrollo actual y cumplimiento de las condiciones; sin embargo, el Ministerio de Educación Nacional identificó ciertas dificultades para la ejecución de la evaluación, que llevaron a la generación de un modelo ajustado de la evaluación de la relación docencia servicio para la ejecución de una prueba piloto que permite identificar la viabilidad técnica y económica que tienen algunas condiciones para ser implementadas por la institución prestadora de servicios de salud y/o Institución de educación superior.

Para lograr lo anterior, es necesario que el estudiante durante el transcurso de la carrera aplique los conocimientos adquiridos en diferentes contextos: las prácticas formativas, los laboratorios de simulación, espacios de aula y de trabajo autónomo, pues en estos espacios regu- lados o no, se pueden identificar las dificultades del estudiante, con el fin de formular estrategias de mejora para conseguir el desarrollo de las competencias planeadas.

Al revisar las características de calidad de las prácticas formativas en enfermería, se sugiere que el estudiante debe recoger las recomendaciones del docente de práctica y de su tutor, dar respuesta a las necesidades de la institución y pacientes, observar las políticas y normas, adherirse a los procesos y protocolos, adaptarse a un clima organizacional, y además, en esta práctica es donde se genera y prueba la validez y calidad de las propuestas teóricas entregadas en la institución de educación superior, las cuales se evidencian a través de una serie de documentos e informes físicos que tan solo el procesamiento de datos conlleva a una inversión de tiempo importante para obtener resultados que permitan la toma de decisiones ${ }^{(1)}$. Se plantea entonces, que el estudiante demostrará el conocimiento y lo aplicará en los escenarios de práctica en un medio controlado, acompañado por los involucrados descritos en el convenio docenciaservicio; el resultado de este engranaje se cree es el reflejo de la calidad del proceso de enseñanza aprendizaje con la interacción de las instituciones de salud, el cual requiere ser evaluado, según las disposiciones de calidad a las que se abocan los programas de educación superior, en especial, los programas del área de la salud, en donde, desde las 
Escobar Escobar MB, Álvarez Castaño N, Rendón Caro S, Villegas Quintero M, García

Gallego V, Mantilla Sánchez LM

obligaciones y cumplimientos, son compartidos Instituciones de Educación Superior (IES), Instituto Prestador de Servicios de Salud (IPS) y los actores del proceso guardan gran relevancia en la evaluación de las prácticas formativas. Es por esto que se debe trascender el diligenciamiento y la adopción de disposiciones de la norma, a convertirlo en un componente valioso de la calidad de los programas que representan más del 60\% de los planes de estudio de los programas de enfermería del país. Se realiza esta investigación en la Universidad Católica de Manizales, durante los años 2019 y 2020, revisando la información de los años 2015 al 2017. Con el fin de resolver la pregunta ¿Cuáles son las condiciones de calidad de las prácticas de VII y VIII semestre del programa de Enfermería de la Universidad Católica de Manizales con relación a los criterios básicos definidos por el Ministerio de protección social, el Ministerio de Educación Nacional (MEN)?

\section{METODOLOGÍA}

El estudio es de tipo descriptivo, retrospectivo y documental.

Población: Formatos de evaluación diligenciados por los involucrados (estudiantes, usuarios, docentes de estos semestres, profesionales de las IPS involucrados en las prácticas) y planes de delegación progresiva de todas las prácticas de 2015 , 2016, 2017 de VII y VIII semestre del programa de Enfermería de la Universidad Católica de Manizales, aplicando las variables de estos instrumentos con el "modelo de evaluación de la relación docencias servicio, criterios básicos desde la calidad de prácticas formativas 2004 del
Ministerio de protección social"(2).Todos estos aspectos evaluados deben reflejar la Política Institucional, incluida en el (Factor 1), los actores en el proceso (Factor II), Campo de actuación (Factor III), las condiciones necesarias (Factor IV) y Relación con el entorno (Factor V).

En la Tabla 1 se describen los factores que se tienen en cuenta para validar la calidad de dichas prácticas.

Criterios de inclusión: Formato de informe final de práctica formativa, plan de delegación progresiva, encuestas de usuarios, profesionales, estudiantes y docentes legibles, debidamente diligenciadas.

Criterios de exclusión: Formatos que no estén legibles y completamente diligenciados.

Criterios de rigor metodológico: Recolección de la información: La técnica de recolección de la información fue revisión documental, de los últimos tres años. Se aplicaron las variables de este modelo que se están implementando en el programa, como acceso de los estudiantes a la práctica, conocimiento de normas de todos los involucrados en las practicas, relaciones interpersonales durante las practicas, promoción de investigación y proyección social durante la práctica ${ }^{(3)}$.

Para la identificación y selección de la metodología e instrumentos, los investigadores tuvieron en cuenta la información suministrada por la literatura científica, el marco de regulación en que operan las prácticas formativas, los factores y las características del modelo de evaluación de las prácticas formativas del Ministerio de Educación Nacional (MEN), la evaluación de la práctica formativa entre los años 2015 al 2017, el comportamiento 
Calidad de las prácticas formativas en los últimos semestres de Enfermería...

de los actores en los diferentes convenios docencia servicio y la contextualización de la evidencia a las circunstancias específicas. Esto con el fin de que esta información ayudara a identificar las condiciones de calidad de tal manera que la orientación de las recomendaciones tuviera el máximo impacto en la entrega de resultados.

Tabla 1. Elementos que componen el modelo de la relación docencia-servicio.

\begin{tabular}{ll}
\hline Factores & Definición \\
\hline 1. Definición de & El objeto de la relación docencia-servicio debe hacer explícito \\
Relación de la & el compromiso de las instituciones participantes en la \\
Docencia-Servicio: & realización de las prácticas formativas, del o de los programas \\
& que conjuntamente se proponen desarrollar, así mismo debe \\
& hacer explícito el alcance de dicha relación, determinando el \\
& conjunto de criterios que sirvan de referencia fundamental sobre \\
& el futuro deseable y posible para la organización yestión de \\
los programas a desarrollar en un periodo definido. & El acceso de estudiantes a las prácticas formativas debe estar \\
Docentes en prácticas & determinado por criterios que definan en cada programa \\
formativas & educativo el número de estudiantes, considerando las \\
& exigencias y características de la calidad de la prestación de \\
servicios, los derechos de los usuarios de dichos servicios y las \\
competencias profesionales a desarrollar por los estudiantes. \\
3. Procesos Académicos & $\begin{array}{l}\text { La definición de los contenidos de las prácticas formativas de } \\
\text { los programas a desarrollar debe ser consistente con los } \\
\text { Formativas }\end{array}$ \\
objetivos educacionales y las competencias profesionales a \\
Administración y & adquirir por los estudiantes \\
La relación docencia-servicio requiere una estructura \\
organizacional definida, que debe responder por la dirección, la \\
planeación, la ejecución, el seguimiento, la evaluación y la \\
retroalimentación, de las actividades que se derivan de los \\
acuerdos entre las instituciones que participan en dicha relación \\
docencia-servicio.
\end{tabular}

Búsqueda y selección de la literatura: Se definieron y describieron los conceptos sobre la búsqueda bibliográfica y su planificación en el contexto del proyecto a partir de la pregunta formulada, con relación a los criterios básicos definidos por el Ministerio de protección social, el Ministerio de Educación Nacio- nal (MEN). Se buscaron las fuentes más relevantes, en las diferentes bases de datos que tiene la Universidad, como Scielo, Hinari, Proquest, Scopus, entre otros. Además de la normativa, los informes de prácticas formativas archiva-dos desde el año 2015 al 2017, junto con informes y documentos propios de la coordinación de 
Escobar Escobar MB, Álvarez Castaño N, Rendón Caro S, Villegas Quintero M, García

Gallego V, Mantilla Sánchez LM

las prácticas, como los informes semestrales, estudios de percepción, convenios, y demás documentos inherentes a las prácticas de enfermería.

Evaluación de la información y síntesis de la evidencia: Para la evaluación de la evidencia científica se definió valorar la información y la clasificación de artículos en términos de calidad, según la importancia de los resultados y la aplicabilidad para las prácticas formativas en enfermería, los artículos que aportaron evidencias de las mejores prácticas, para ser tomadas en cuenta en el proyecto y resultados de investigación de prácticas similares

Se aplicó el instrumento definido en el Modelo de evaluación del Ministerio de Educación en Colombia a las fuentes sugeridas para la evaluación de criterios estándares básicos de calidad y se revisaron los informes de prácticas que cumplieran con los parámetros establecidos. Análisis de conveniencia ${ }^{(4)}$.

El estudio fue documental, por lo tanto no se interactuó con la población directamente; desde la parte ética es de bajo riesgo por no tener intervención directa.

\section{RESULTADOS}

Se realizó el análisis de los aspectos verificados y hallazgos encontrados en cada uno de los estándares inspeccionados.

La característica uno sobre el objeto y alcance de la relación docenciaservicio muestra cómo la expresión del objeto de relación es clara en las instituciones participantes de las prácticas formativas del programa de enfermería, y a través de los responsables del convenio es posible la organización y gestión de los compromisos programados para desarrollarse en un periodo definido. Algunas instituciones, sobre todo las acreditadas como IPS universitaria, tienen mejores procesos y personal para hacer seguimiento.

Se descubre que la UCM cumple con todos los criterios de idoneidad como institución educativa y asimismo es exigente al hacer el acercamiento a las instituciones prestadoras de servicios de salud, para participar en la relación docencia-servicio; busca dejar todos los aspectos de las prácticas formativas explícitos y relacionados con el desarrollo del programa, lo que le da viabilidad al cumplimiento del objeto de la relación docencia- servicio. A través de los comités docencia-servicio (CODAS) se asegura la interrelación de todos los procesos, incluyendo la evaluación del convenio, por parte de los diferentes actores que participan en el proceso.

Todos estos requisitos son tenidos en cuenta en los documentos presentados en diferentes instituciones, igualmente para la planeación y para la distribución de los estudiantes, todo se lleva a cabo en concertación con el director de programa, coordinador de prácticas formativas y los docentes tutores.

Lo anterior no sería posible sin contar con los anexos técnicos que contemplan las competencias que deben desarrollar los estudiantes. Estos luego están inmersos en "los planes de práctica" los cuales se hacen llegar a cada institución donde se realice la práctica formativa. Contienen una información general del semestre, con fecha de inicio y finalización, información específica de la práctica o rotación, nombre de los 
Calidad de las prácticas formativas en los últimos semestres de Enfermería...

estudiantes en rotación y docente, horarios, objetivos educacionales de la rotación o la práctica, el sistema de evaluación de las prácticas formativas, evaluación de la práctica, plan de delegación progresiva y demás documentos exigidos por la institución donde realiza la práctica formativa.

Documentos existentes en el sistema integrado de gestión (SIG). Cada institución posee el documento "Información específica de las prácticas formativas, por escenario", definido por el Ministerio de Educación y firmado por ambas partes para dar claridad a las competencias que debe alcanzar el estudiante.

Los resultados recopilan la información de treinta y tres IPS con las cuales se tiene convenio docencia-servicio durante el periodo evaluado. Asimismo, los resultados se muestran en gráficos radiales, los cuales permiten describir valores relativos porcentuales a un punto central, cuando las categorías no son comparables directamente.

La primera variable hace referencia al acceso de estudiantes a la práctica formativa, donde se evalúan ocho preguntas de medición, con respuesta: $\mathrm{Si}$, No y En Parte.

La Figura 1 corresponde a las respuestas positivas por parte de los estudiantes (35 por año) a las preguntas de medición, se observa un nivel de afirmación por encima del $80 \%$, donde el año 2017 es el que muestra los mejores indicadores con respecto al promedio general, mientras que para el año 2016, siete de las ocho preguntas de medición se encontraron por debajo de este porcentaje.

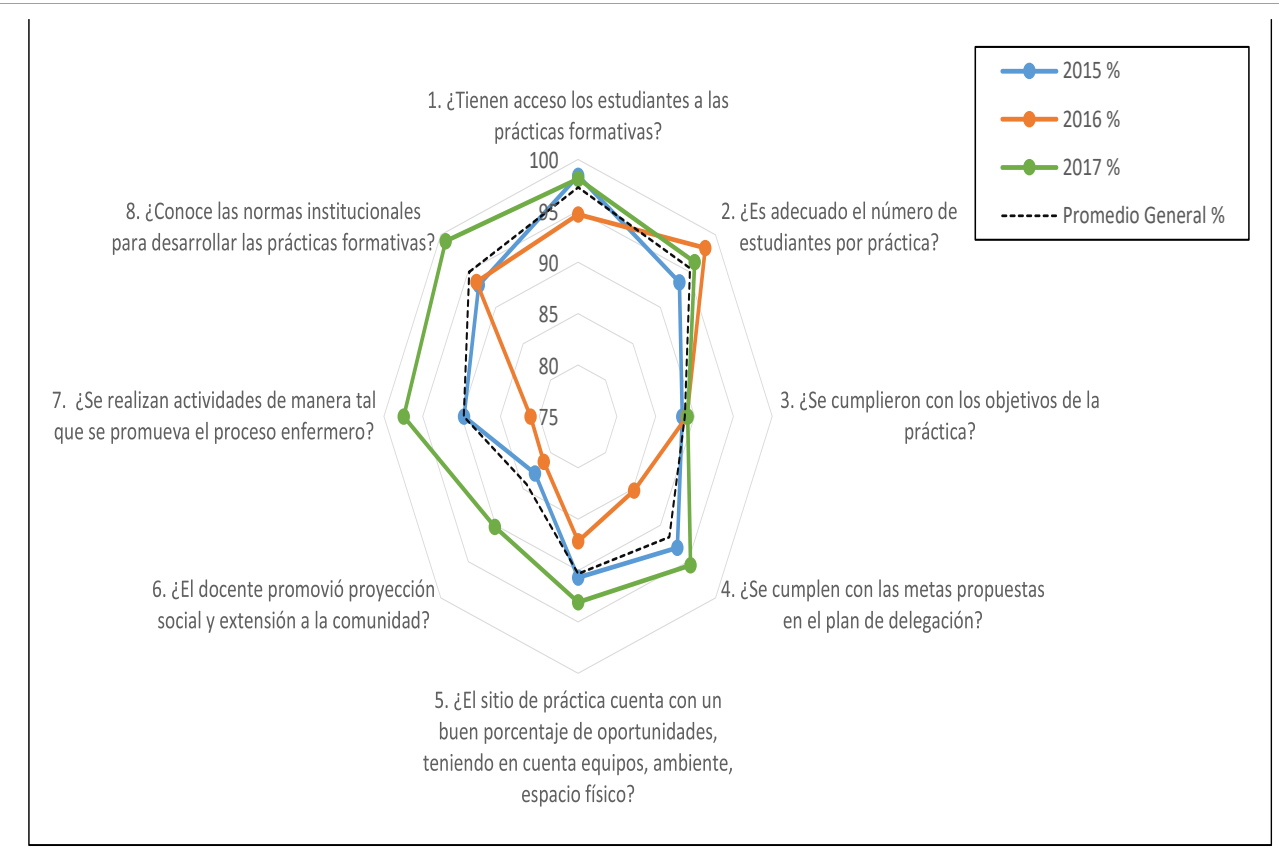

Figura 1. Respuestas afirmativas a las preguntas de medición de la categoría Acceso de estudiantes a las prácticas formativas. Fuente: Elaboración propia. 
Escobar Escobar MB, Álvarez Castaño N, Rendón Caro S, Villegas Quintero M, García

Gallego V, Mantilla Sánchez LM

En los resultados para las mismas ocho preguntas de medición, hechas a los estudiantes de las prácticas formativas, la mayoría de las preguntas tienen valores porcentuales por debajo del 3\%, sin embargo, para el año 2015 y 2016 se presentaron los valores porcentuales más altos con un $7,7 \%$ y $9,4 \%$, respectivamente.

Cabe resaltar, además, que los semestres evaluados tienen dentro de las competencias a desarrollar, procesos administrativos en manejo de personal, toma de decisiones, planeación, seguimiento a la mejora continua, programas de seguridad de paciente, condiciones de habilitación o acreditación, componentes del sistema obligatorio de garantía de calidad, para lo cual los estudiantes realizan un plan de mejoramiento en séptimo y un proyecto en octavo semestre.

Las demás actividades que están contempladas en el plan de delegación, recogen las actividades del día a día, en atención directa a paciente, manejo de personal, educación a usuarios familia y comunidad, sistema de información y atención al usuario, caracterización del riesgo, las cuales son las que no se logran completamente por la gestión realizada en la parte administrativa.

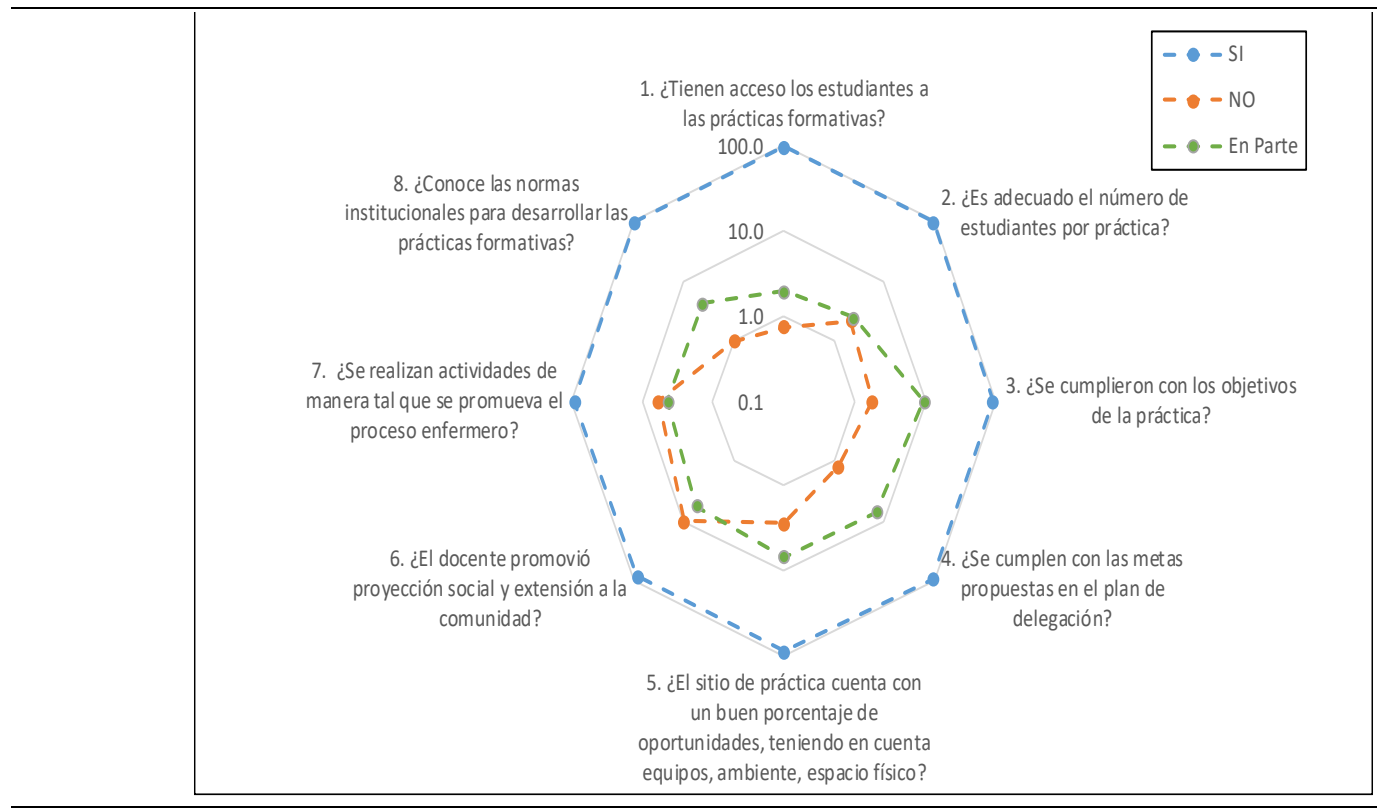

Figura 2. Promedio general de respuestas a la categoría "Acceso de estudiantes a las prácticas formativas”. Fuente: Elaboración propia.

En cuanto al comportamiento de los promedios generales, para los tres años evaluados con relación a las tres posibles respuestas, la Figura 3 muestra una evidente satisfacción general a las ocho preguntas de medición, mientras que las respuestas "no" y "en parte" reflejan hete- rogeneidad de percepciones, pero nunca estando por encima del 10\%. Estos resultados reflejan que la calidad en el acceso a los estudiantes, para desarrollar sus competencias, está dada en alto grado.

Para la segunda y tercera variable se evalúan siete preguntas de medición, 
Calidad de las prácticas formativas en los últimos semestres de Enfermería...

con respuesta: "Si", "No" y "En Parte" que hacen referencia a los profesores de la entidad educativa y al personal de salud de la institución prestadora de servicios que participan en el convenio Docencia Servicio.

Los resultados de estas categorías se presentan en conjunto con el fin de evidenciar de forma comparativa la percepción tanto de profesores como de profesionales, así mismo, los valores reflejan el promedio general para el periodo evaluado en cada una de las posibles respuestas.
Durante la práctica del semestre se realizan rotaciones guardando correspondencia con los componentes teóricos, competencias, habilidades y destrezas de cada asignatura, planteados en los Proyecto Académico de Asignatura (PAA) y en el plan de delegación progresiva. La práctica integral de gestión del cuidado de VIII semestre se desarrolla con dos semanas teóricas de inducción, y quince (15) semanas de prácticas.

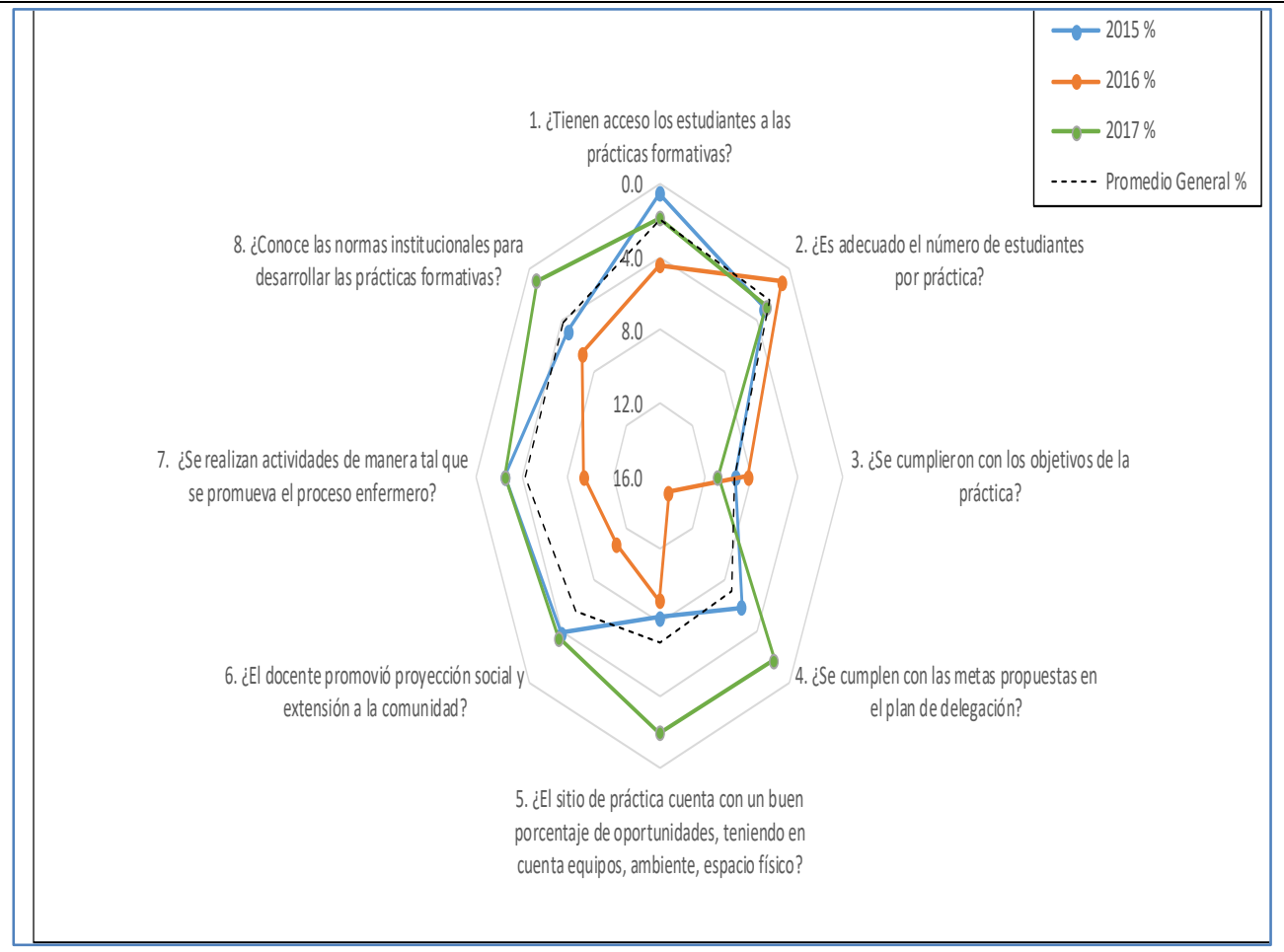

Figura 3. Respuestas "en parte" a las preguntas de medición de la categoría "Acceso de estudiantes a las prácticas formativas". Fuente: Elaboración propia.

Para determinar el cumplimiento de la característica acceso de estudiantes a las prácticas formativas, es importante decir que los anexos técnicos cumplen con todos los criterios explícitos para los estudiantes y docentes. Se tienen varios mecanismos para determinar su acceso, el primero son los anexos técnicos que determinan el número de estudiantes en dichas prácticas, en relación con los criterios definidos para la admisión. 
Escobar Escobar MB, Álvarez Castaño N, Rendón Caro S, Villegas Quintero M, García

Gallego V, Mantilla Sánchez LM

\section{DISCUSIÓN}

La evaluación de la percepción de las prácticas es un proceso humanizado que apunta a la excelencia como criterio de calidad humana. Los aspectos referidos señalan que las prácticas en el programa de enfermería han sido de gran utilidad para favorecer una cultura que se encuentra en marcha, buscando ante todo perfeccionar las competencias, capacidades y actitudes de los estudiantes como el talento humano, indispensable para ayudar en el cumplimiento de las metas institucionales de los sitios de práctica. Afianzar los pasos que se dan hacia la construcción de esta cultura "reconoce que la evaluación educativa cumple, cuando menos, tres funciones básicas: diagnosticar, juzgar y mejorar"(5).

El diagnóstico, la elaboración argumentada de juicios y la disposición hacia la mejora continua se constituyen en una triada potente que robustece en la institución el cumplimiento de sus objetivos misionales, acercándola sólidamente hacia el logro de sus metas de calidad como institución que forma para la vida y de manera sostenible. En el programa de enfermería, a la luz de los hallazgos de la investigación, se comprueba que evaluar supone siempre el acto de establecer el valor de algo y, para ello, no bastará con la mera recogida de información, sino que es necesario interpretarla, ejercer una acción crítica sobre la misma, buscar referentes, analizar alternativas, ofrecer visiones no simplificadas de los aprendizajes evaluados, entre otras. Evaluar implicará por encima de todo, la creación de una cultura evaluativa donde ubicar adecuadamente este tipo de conocimiento ${ }^{(6)}$.

Las prácticas, como instrumento, son para el programa de enfermería un elemento que potencia la cultura de la acción y genera una expansión significativa de las estrategias y prácticas organizacionales de la universidad, que como empresa educativa tiene, en el fondo de su misión, la pretensión de contribuir en el logro de calidad humana en todos sus actores y profesionales. "Esto significa que todos los esfuerzos económicos, realizados por las empresas que desean y requieren ser responsables socialmente, serían en vano, si sus colaboradores no están inmersos en una actitud de calidad humana"(7,8).

La práctica adquiere una configuración valiosa desde el punto de vista de la responsabilidad social de la enfermería, porque no solo se concibe como un programa que forma a los nuevos profesionales en este campo, si no que la institución se proyecta hacia las comunidades que ven en el saber enfermero una mediación científica y socio cultural que mejora las prácticas asistenciales y coadyuvan en la gestión humanizadora del cuidado. Este planteamiento que se deriva del análisis comprensivo de la evaluación docente, remite a ponderar que, en cualquier circunstancia, el enfermero que prepara y capacita a los nuevos profesionales en esta rama del conocimiento debe acentuar la vida como un valor supremo que garantiza la calidad de cualquier servicio que se le preste a los seres humanos, por cuanto "preservar la calidad en salud es vital; para toda organización es darle vida a la misma, que perdure en momentos difíciles que se le presenten, debe solventar estos inconvenientes porque su objetivo es preservar la vida de los demás con sus servicio $^{(9,10)}$. 
Calidad de las prácticas formativas en los últimos semestres de Enfermería...

La calidad de la práctica formativa constituye el nivel más elevado del Sistema Nacional de Educación y tiene como objetivo central la formación académica y la superación profesional continua de los estudiantes de pregrado durante su vida profesional, lo que contribuye de forma sistemática a la elevada productividad, eficiencia y calidad del trabajo. La obtención de resultados significativos está en correspondencia con el trabajo sostenido en este proceso, siguiendo el patrón de organismos externos encargados de evaluar y acreditar la calidad educativa en la educación superior $^{(11,12)}$.

Al respecto, Mondragón ${ }^{(13)}$, señala que surgió, debido a factores relacionados con el crecimiento y expansión del servicio educativo a partir de la década de 1960; sin embargo, es a finales de la década de 1980, y en el transcurso de la siguiente década, cuando los gobiernos de los países en América Latina prestan interés sobre la temática de la calidad educativa en el nivel superior; verificar los cumplimientos de la calidad para acreditación es una tendencia mundial y en especial, la acreditación de la educación superior, es una tendencia mundial acreditación de la calidad ante organismos externos $^{(14)}$. Sin embargo, aunque en determinados casos esto ha contribuido a mejorar la formación de los estudiantes y el desempeño en proyectos sociales y científicos, se tiende a abordar desde lo administrativo, sin tocar la transformación de los procesos curriculares, las prácticas de aprendizaje, realizando la simulación en los procesos de evaluación de la calidad $^{(15,16)}$.
Los docentes reconocen que la práctica para el estudiante puede servir para prevenir las consecuencias indeseadas del mismo. Esto es similar a la respuesta a las pruebas de altas consecuencias, descrita por Nichols ${ }^{(17)}$, donde los docentes harán "todo lo necesario para cumplir sus metas, no obstante, las prácticas de docencia-servicio actúan en consonancia con los valores de los estudiantes, en la medida que les permite conseguir un trabajo que sea importante. Es necesaria la evaluación de las experiencias que se viven en los campos de práctica, el establecimiento de indicadores de calidad para evaluar prácticas educativas que permite poner el foco en ciertos ítems a los que habrá que prestar especial atención ${ }^{(18,19)}$. El impacto en indicadores de calidad de la atención hospitalaria, se debe realizar partiendo de la identificación de problemas relacionados con: la formación en salud en Colombia, inclusión del componente social en las prácticas formativas, gestión administrativa, modelo de evaluación y financiamiento de la relación docenciaservicio y gestión del conocimiento y de la investigación. De acuerdo con lo planteado por Hurtado ${ }^{(20)}$, es necesario que los docentes, demuestren un nivel de reflexión crítica sobre su propia práctica, tendiente a la mejora continua, por-que existirían aprendizajes de calidad, los que serían bien valorados por los estudiantes.

Es importante la realización de esta reflexión de los sitios de práctica, ya que aquellas del tipo formativas se encuentran diseñadas desde diferentes modalidades que permiten el desarrollo de las competencias, para brindar un cuidado efectivo, previo abordaje de los compo- 
Escobar Escobar MB, Álvarez Castaño N, Rendón Caro S, Villegas Quintero M, García

Gallego V, Mantilla Sánchez LM

nentes teóricos básicos, con sus respectivas aplicaciones en el laboratorio de simulación clínica. Esto lo reafirma Fernández y Torres $^{(21)}$, quienes señalan que entre los principales factores para proporcionar buenas prácticas se encuentran: favorecer el trabajo personalizado; favorecer la comunicación con los estudiantes y estimular el aprendizaje o la adquisición de conocimientos. Giler, Zambrano, Velásquez y Vera $^{(22)}$, refieren que resulta imprescindible promover apoyos y estímulos a los docentes para el desarrollo de unas tareas cada vez más diversas y complejas. Una forma de potenciar innovación y desarrollar métodos centrados en el aprendizajes significativos $^{(23)}$, es mediante la difusión de buenas prácticas y de establecer las condiciones necesarias para que éstas puedan surgir o potenciarse, tal como lo señalan Mas y Olmos ${ }^{(24,25)}$. Aquí es cuando estudios como el descrito, cobran relevancia para difundir los factores que potencian las buenas prácticas. Se puede inferir que la forma de realizar la docencia, es decir las estrategias docentes, las características de los profesores en el aula, así como los buenos campos de práctica, se convierten en los principales factores para potenciar la calidad en la educación, así como los procesos de mejora de la docencia y de las prácticas educativas y, por otro lado, el sentido de involucramiento de los alumnos con su formación, dándole, en general, un enfoque de calidad y de compromiso con la formación de los maestros del siglo $\mathrm{XXI}^{(26,27) \text {. }}$

Es importante tener en cuenta que el programa desarrolla sus prácticas en escenarios clínicos, no clínicos, de acuerdo al planteamiento de las asignaturas. Para la evaluación y control de las prácticas formativas, el programa se ha preocupado por realizar trazabilidad de los documentos tales como: anecdotarios, bitácoras, plan diario de actividades, planes de delegación, encuestas, entre otros; que evidencian el desarrollo de las actividades y procedimientos realizados por los estudiantes en los turnos asignados. El plan de delegación permite identificar las competencias y adquisición de habilidades de cada estudiante por cada rotación programada, esto denota un seguimiento riguroso de los aprendizajes en el escenario práctico, además de ofrecer información valiosa del impacto de la práctica formativa en las instituciones de salud. Pero, como refiere Ramos ${ }^{(28,29,30)}$, existe cierta descoordinación entre la universidad y los centros de prácticas. Los problemas a la hora de seleccionar los centros, tal como explica Fernández ${ }^{(31,32)}$, vuelven a ser un punto importante a considerar, en futuros proyectos de mejora.

\section{CONCLUSIONES}

En conclusión, describir las condiciones de calidad de las prácticas de VII y VIII semestre, del programa de Enfermería de la Universidad Católica de Manizales, con relación a los criterios básicos definidos por el Ministerio de Protección Social, el Ministerio de Educación Nacional (MEN), con relación a los definidos por el programa de Enfermería de la UCM en los años 2015 - 2017, muestra que calidad, calidez y humanización, se entrelazan significativamente para mostrar que la evaluación de la percepción de las prácticas en enfermería es un instrumento que potencializa sus capacidades, 
Calidad de las prácticas formativas en los últimos semestres de Enfermería...

actitudes, aptitudes, talentos y habilidades para lograr en ellos un compromiso ético y axiológico con la excelencia.

El espacio utilizado para la realización de las prácticas son las Instituciones Prestadoras de Servicios de Salud; deben ser lugares que faciliten a los estudiantes encontrar una relación integral entre la formación teórica y su aplicación a la práctica profesional; de esta manera el estudiante podrá incrementar su preparación específica.

Es importante diseñar un adecuado y efectivo mecanismo de seguimiento y control, donde participen todos los actores de este proceso y se puedan introducir los cambios de una manera efectiva $y$ oportuna, sin que esto afecte la calidad del servicio y el nivel académico de los diferentes programas de educación.

La evaluación de las condiciones de calidad existentes, basadas en la documentación de los informes finales de práctica, es una actividad que se debe realizar cada año, como mínimo para no solo ayudar a los sitios con los que se tienen convenio, sino también para mejorar las prácticas de los estudiantes en formación.

Se deja planteada una estrategia educativa en una segunda fase, que impacte para los involucrados en las prácticas formativas.

\section{AGRADECIMIENTOS}

Al Comité Académico y docente de Enfermería y de los centros de práctica por la constancia en llenar los informes y formatos requeridos. A la Dirección de la Universidad Católica de Manizales, por su apoyo y exigencia en el desarrollo de la misma. A los Estudiantes, que representan la materia prima fundamental de nuestra labor, a las entidades de los convenios docencia servicios por su seriedad, responsabilidad, constancia y compromiso con el desarrollo de la Educación de la Universidad y la sociedad.

\section{CONFLICTO DE INTERESES}

Las autoras declaran no tener conflictos de intereses.

\section{REFERENCIAS BIBLIOGRÁFICAS}

1. Espinel A. La tecnología en el aprendizaje del estudiantado de la Facultad de Ciencias Químicas: Universidad Central del Ecuador. Actualidades Investigativas en Educación. 2020; 20(2): 308-347.

2. Organización Panamericana de la Salud /Organización Mundial de la Salud, y Programa de Recursos Humanos. Modelo de evaluación de la relación docencia-servicio criterios básicos de calidad para centros de prácticas formativas. Programa apoyo a la reforma en salud. [Internet] 2004. [Consultado 3 Jul 2021]. Disponible en:

http://www.unipamplona.edu.co/unip amplona/portalIG/home_54/recursos/ 01general/04122012/mod_relacion_d ocencia_servicio.pdf

3. López P. Población muestra y muestreo: Punto Cero. 2004; 09(08): 69-74.

4. Apodaca O, Ortega P, Verdugo B, Reyes B. Modelos educativos: Un reto para la educación en salud. Ra Ximhai. 2017; 13 (2): 77-86.

5. Torres P. ¿Qué estamos haciendo en Cuba en evaluación educativa? Revista Iberoamericana de 
Escobar Escobar MB, Álvarez Castaño N, Rendón Caro S, Villegas Quintero M, García

Gallego V, Mantilla Sánchez LM

Evaluación Educativa. [Internet]. 2016 [Consultado 3 Jul 2021]. Disponible en: https://revistas.uam.es/index.php/riee /article/view/4685

6. Marzal M, Borges J. Modelos evaluativos de Metaliteracy y alfabetización en información como factores de excelencia académica. Revista española de documentación científica. 2017; 40(3): e184-e184.

7. Anatb A, Rector R, Leite E. El emprendimiento ¿actitud o como competencia del capital humano? Una mirada conceptual desde la universidad de Holguín, Cuba. Holos. 2018; 8, 109-137.

8. Amariles A. La humanización, el cuidado humanizado y la formación ciudadana: reconocimiento del desarrollo formativo en el programa de enfermería de la universidad de caldas. repositorio UCM. 2017. 153.

9. Antúnez S, Fernández Ll. Delgado F. Calidad, medio ambiente, seguridad y salud, y control interno en el contexto económico actual: diagnóstico de un laboratorio farmacéutico cubano. Cofin Habana. 2017; 11(1): 1-12.

10. Escobar-Castellanos B, CidHenríquez P. El cuidado de enfermería y la ética derivados del avance tecnológico en salud. Acta bioethica. 2018; 24(1): 39-46.

11. Consejo Nacional de Acreditación. Lineamientos para la acreditación de programas. Corcas editores Ltda. 2006. [Internet] 2006 [Consultado 3 Jul 2021]. Disponible en: https://www.cna.gov.co/1741/articles -186359_lineamientos_2.pdf
12. Consejo Nacional de Acreditación. Lineamientos de Acreditación-CNA. [Internet] 2016 [Consultado $3 \mathrm{Jul}$ 2021]. Disponible en: https://www.cna.gov.co/1741/article186359.html.

13. Mondragón $\mathrm{H}$ y Rosa A. La acreditación y certificación en las instituciones de educación superior. Hacia la conformación de circuitos académicos de calidad: ¿exclusión o integración? [Internet] 2006 [Consultado 3 Jul 2021]. Disponible en:

https://doi.org/10.26457/recein.v7i26 .240

14. Zambrano S, Gordillo O, Perlaza M. Evaluación de un programa de proyecto de vida a través de la controversia. Revista Virtual Universidad Católica del Norte. 2019; 0(57): 60-72.

15. Escobar M B. La evaluación por competencias en los escenarios emergentes de la realidad. 2017.

16. Martínez Zamora, Marco. La evaluación de la práctica docente un modelo de integración de las dimensiones cuantitativas y cualitativas. 2016. Debates en Evaluación y Currículum/ Congreso Internacional de Educación Evaluación Año 2, No. 2, Septiembre a Agosto de 2017.

16. Nichols S, Brewington S. Preservice teachers' beliefs about high-stakes testing and their working environments. Education Policy Analysis Archives. 28(0):30. [Internet] 2020 [Consultado 3 Jul 2021]. Disponible en: 
Calidad de las prácticas formativas en los últimos semestres de Enfermería...

https://doi.org/10.14507/epaa.28.487

7

17. Saif M, Fernández Y. Indicadores de evaluación del impacto de la gestión académica de posgrado para la calidad de la formación pedagógica universitaria. Opuntia Brava; 10(2): 130-143. [Internet] 2018 [Consultado 3 Jul 2021]. Disponible en: https://doi.org/10.35195/ob.v10i2.93

18. Escobar M, Escobar R. Propuesta de un marco de trabajo transdisciplinario para la práctica asistencial en el área de la salud. Manizales, Colombia: Universidad Católica de Manizales. 2018.

19. Hurtado M, Bueno A, Martínez O. Percepción del alumnado sobre la utilidad de las actividades de aprendizaje para desarrollar competencias. Revista de Investigación Educativa.; 34(1): 221240. [Internet] 2016 [Consultado 3 Jul 2021]. Disponible en: https://doi.org/10.6018/rie.34.1.2251 31

20. Fernández B, Torres G. Actitudes docentes y buenas prácticas con TIC del profesorado de educación permanente de adultos en Andalucía. [Internet] 2015 [Consultado 3 Jul 2021]. Disponible en: https://doi.org/10.5209/rev_RCED.20 15.v26.43812

21. Giler L, Zambrano M, Velásquez S, Vera M. Padlet como herramienta interactiva para estimular las estructuras mentales en el fortalecimiento del aprendizaje. Dominio de las Ciencias. 2020; 6(3): 1322-1351.
22. López B, Esteban P, Mateo P, Peleato I, Rodríguez M. Métodos centrados en el aprendizaje, estrategias y enfoques de aprendizaje en estudiantes universitarios. Revista de educación. 2015; 370: 229-254.

23. Mas T, Olmos R, Mas T, Olmos R. El profesor universitario en el espacio europeo de educación superior: La autopercepción de sus competencias docentes actuales y orientaciones para su formación pedagógica. Revista Mexicana de Investigación Educativa. 2016; 21(69): 437-470.

24. Rodríguez N, Gutiérrez M, Wong O, López C. Eficiencia académica: Un indicador del que se requiere conocer más. EDUMECENTRO. 2015; 7(3): 188-194.

25. Yáñez G, Soria B. Reflexión de buenas prácticas docentes como eje de calidad en la educación universitaria: Caso escuela de ciencias empresariales de la universidad católica del norte. Formación universitaria; 10(5): 59-68. [Internet] 2017 [Consultado 3 Jul 2021]. Disponible en: https://doi.org/10.4067/S071850062017000500007

26. Suárez D. Docentes, relatos de experiencia y saberes pedagógicos. La documentación narrativa de experiencias en la escuela. Investigación Cualitativa. 2017; 2(1): 42-54.

27. Rodríguez N, Gutiérrez M, Wong O, López C, Eficiencia académica: Un indicador del que se requiere conocer más. EDUMECENTRO. 2015; 7(3): 188-194. 
Escobar Escobar MB, Álvarez Castaño N, Rendón Caro S, Villegas Quintero M, García

28. Ramos E. Análisis de aplicación de la responsabilidad civil médica en los convenios docencia-servicio por daño en el paciente provocado por estudiantes en Colombia. [Tesis]. Escuela de Derecho y Ciencias Políticas. 2021.

29. Seidmann S. Identidade pessoal e subjetividade social: Educação e constituição subjetiva. Cadernos de Pesquisa. 45(156): 344-357. [Internet] 2015 [Consultado 3 Jul 2021]. Disponible en: https://doi.org/10.1590/19805314320 4
Gallego V, Mantilla Sánchez LM

30. Cortés B. La evaluación formativa, un camino por recorrer en la práctica docente. Enfermería Universitaria. 2008; 5(3): 31-36.

31. Fernández N. Evaluación y mejora del prácticum en las titulaciones de Ciencias de la Educación de la UPV/EHU. Revista de Psicodidáctica;11(1), Article 1. [Internet] 2006 [Consultado $3 \mathrm{Jul}$ 2021]. Disponible en: https://ojs.ehu.eus/index.php/psicodid actica/article/view/349 